\title{
Bauhinia Variegata VAR. CANDIDa (Caesalpinioideae, Cercideae), NueVa adVenticia en las Yungas de Argentina
}

\author{
MARÍA A. ZAPATER y EVANGELINA C. LOZANO
}

\begin{abstract}
Summary: Bauhinia variegata var. candida (Caesalpinoideae, Cercideae), new adventitious species in Argentinean Yungas. This paper includes the first record of adventive population of Bauhinia variegata var. candida in Argentinean Yungas. The specimens collected in Oran, Salta Province, in the Selvas Pedemontanas, are described and illustrated.
\end{abstract}

Key words: Bauhinia, Caesalpinoideae, adventitious flora, Yungas, Argentina.

Resumen: Este trabajo incluye el primer registro de una población adventicia de Bauhinia variegata var. candida en las Yungas de Argentina. Se describen e ilustran los ejemplares recolectados en las Selvas Pedemontanas de Orán, provincia de Salta.

Palabras clave: Bauhinia, Cesalpinoideae, flora adventicia, Yungas, Argentina.

Bauhinia L. es un género pantropical y subpantropical, que reúne unas 300-350 especies en su amplia circunscripción, muy variables morfológicamente y en tipos de hábitat (Sinou et al., 2009), distribuidas en América, Asia, África y Oceanía (Gamberro \& Fortunato, 2001). En América se encuentra desde México hasta el norte de Argentina (Lewis \& Forest, 2005).

En Argentina el género alcanza su distribución más austral o marginal, con siete especies y una variedad (Fortunato, 1986, 1997), que habitan en las provincias de Jujuy, Salta, Formosa, Corrientes y Misiones. Además, se cultivan extensamente dos especies, una nativa y otra exótica, con fines ornamentales y de arbolado urbano (Fortunato, 2002). Bauhinia variegata L. con sus dos variedades, la var. típica y la var. candida Voigt., es la especie exótica ampliamente cultivada en la región noroeste y central. Recientemente se ha encontrado una población de $B$. variegata var. candida en la Selva Pedemontana de las Yungas en el departamento Orán, al norte de Salta, en un área

\footnotetext{
${ }^{1}$ Facultad de Ciencias Naturales, Universidad Nacional de Salta, Avda. Bolivia 5150, 4400 Salta, Argentina; aliciazapater@yahoo.com.ar (autor corresponsal).
}

fronteriza con Bolivia. Se describe e ilustra este nuevo registro para la flora adventicia de Argentina.

Bauhinia variegata $L$. var. candida Voigt., Hort. Suburb. Calcutt.: 253. 1845 (Figs. 1, 2).

B. candida Roxb., Fl. Ind. 2: 318-319. 1832, nom. inval.

Árbol inerme de 5-10 m alt. y 0,20 m diám.; corteza grisácea, longitudinalmente rugosa. Hojas bilobadas, regularmente ovadas, hasta de $12 \mathrm{~cm}$, coriáceas, ápice obcordado a hendido, base cordada o redondeada, raro obtusa, glabras. Inflorescencia pauciflora, abreviada, en racimos extraxilares. Botón floral ovado-fusiforme, con largo estrechamiento basal correspondiente al hipanto, ápice gradualmente aguzado. Hipanto de 1,5-2 x 0,2-0,25 cm, carnoso, hueco, largamente tubular, costillado, densamente tomentoso. Cáliz de 2-3,2 cm, espatáceo, pubérulo y con tricomas glandulares anaranjados regularmente esparcidos; pétalos desiguales de 3,5-5 x 1,3-2,5 cm, blancos, cortamente unguiculados; solo el principal con nervios verde-amarillentos notables, ápice agudo hasta cortamente acuminado; estambres tres largos y dos cortos, de 2,4-3,5 $\mathrm{cm}$ y $1,5-2,5 \mathrm{~cm}$ respectivamente, a menudo con algún estaminodio 
Bol. Soc. Argent. Bot. 48 (3-4) 2013

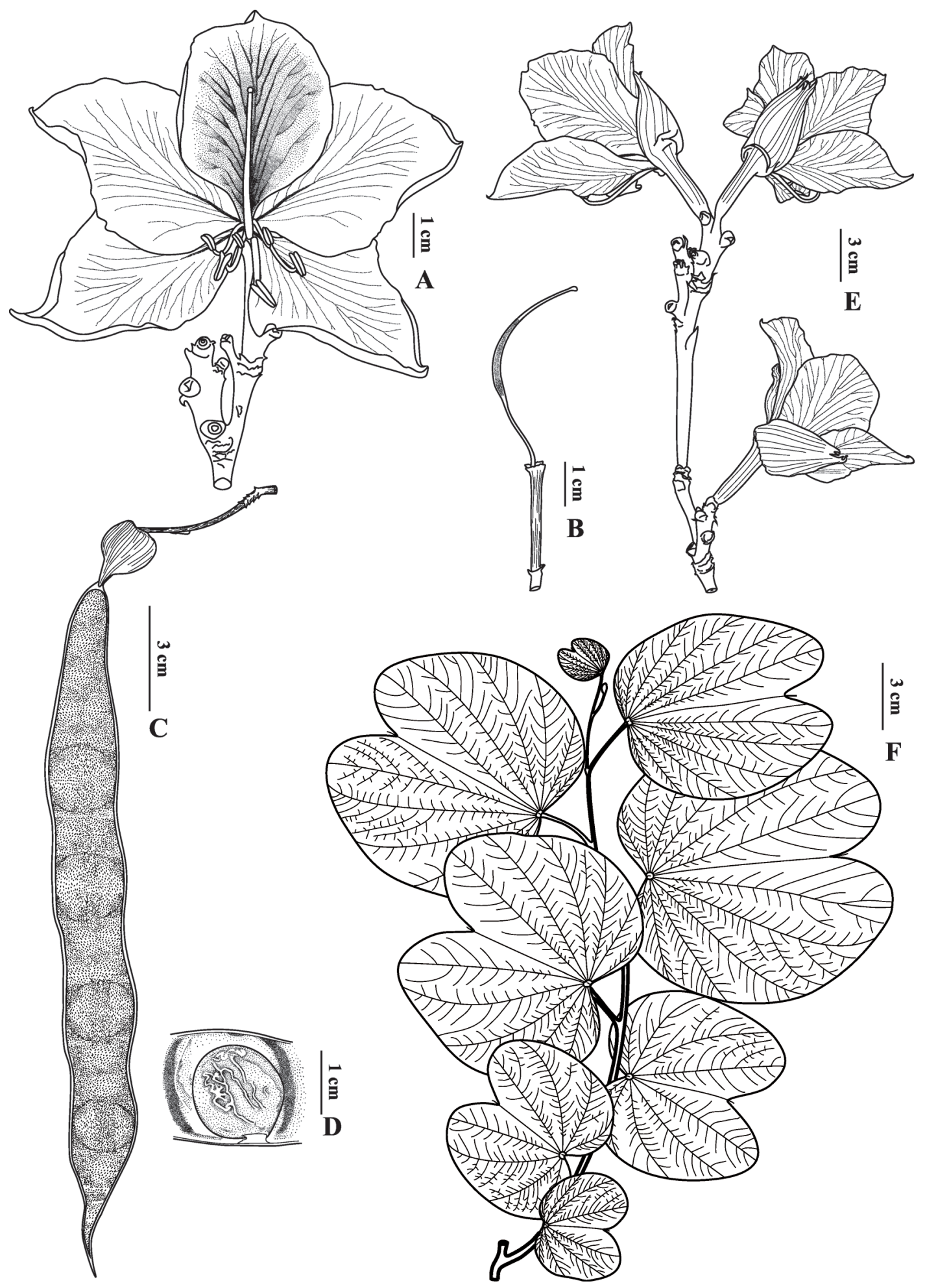

Fig. 1. Bauhinia variegata L. var. candida. A: Flor. B: Gineceo. C: Fruto. D: Semilla. E: Rama florífera. F: Rama vegetativa. De Zapater et al. 2855 (MCNS). 
capilar; anteras dorsifijas de 5-7 mm. Ginóforo de 3-3,3 x 0,1 cm, excéntrico, unilateralmente adnato al hipanto; gineceo falcado, ovario de 1,2$1,3 \times 0,12-0,15 \mathrm{~cm}$, lateralmente comprimido, con tricomas lanosos y escamiformes en forma de lágrima en las caras; estilo erguido de 1,1-1,4 cm, lateralmente pubescente. Legumbre coriácea de 15$22 \times 1,5-1,9 \mathrm{~cm}$, castaña, oblicuamente reticulada, largamente apiculada, glabra; semillas redondeadas de hasta $1,5 \mathrm{~cm}$ diám., castañas, comprimidas, con arilo de $3 \times 1 \mathrm{~mm}$.

Nombres vulgares. Árvore de Sao Thomaz blanco; mountain ebony.

Material estudiado. ARGENTINA. Prov. Salta: Dpto. Orán, Ruta Nacional 50, antes de la Aduana y Puente Internacional del Río Bermejo, frontera con Bolivia, a la derecha de la Ruta, 13-X-2009 (fl, fr), Zapater et al. 2855 (MCNS, SI); del mismo lugar, 17-XII-2009 (fr), Zapater et al. 2866 (MCNS); del mismo lugar, 19-X-2010 (fl, fr), Zapater et al. 3004 (MCNS).

Obs. La población encontrada es monoespecífica, densa y los numerosos ejemplares que la componen poseen flores blancas con el pétalo superior con nervios verde-amarillentos, lo que la diferencia claramente de la var. típica con pétalos rosados a rojizos (eventualmente blancos) siempre con manchas o puntos rojos y amarillos y el pétalo superior más vistoso por la intensa coloración de los nervios. Una característica interesante de la var. candida en la población estudiada es que el androceo posee estambres de desigual longitud, tres largos y dos cortos, lo que no se encontró mencionado anteriormente para la especie; también es particular el indumento del cáliz, hipanto y gineceo. Además se ha comprobado en esta variedad, la reducción total o parcial de los 5 estaminodios capilares que se encuentran descriptos por Fortunato (2002) para la especie.

La existencia de esta población muestra que $B$. variegata var. candida puede comportarse como pionera y permite suponer que podría colonizar claros de la Selva Pedemontana producidos por efectos antrópicos, muy frecuentes en este ambiente debido a su accesibilidad; lo que se encontraría facilitado por la elevada producción de semillas. La variedad ya ha sido citada como invasora en África (Biegel, 1977).

Se revisó el holotipo de $B$. candida Aiton depositado en el herbario BM, que fuera designado basónimo de B. variegata var. candida, constatándose que dicho ejemplar no es el tipo de la variedad, lo que concuerda con las características de la descripción original de Aiton (Hort. Kew. 2: $49,1789)$ y que fuera previamente indicado por Voigt (1845) al realizar la combinación actualmente vigente.

La var. candida fue inicialmente denominada por Roxbourgh (1832) como B. candida, nombre que resultó invalidado por la existencia de $B$. candida Aiton, que corresponde a una especie diferente. Posteriormente, Roxbourg al comparar B. candida con B. variegata L. (Wight \& Walker-Arnott, 1834), menciona que "solamente podía ser una variedad de la última por la coloración de las flores con cuatro pétalos blancos y el quinto variegado en el haz con amarillo y verde". En esta obra también se menciona al menos un sintipo? proveniente de Bengal, India, Wall. L. n. 5796 (ex Roxb. Herb.), de la Lista de Wallich (1828), ejemplar que no pudimos localizar en BM, K, LINN (com. pers. L. Brooks, G. Lewis y J. Hunnex, 2012). En 1845, Voigt establece la combinación $B$. variegata var. candida, en base a $B$. candida Roxb. (no a Aiton) y a las colecciones marcadas en Wallich (1828) con localización en Bengal, (Serampore) Kuvarikot, Islamnaghur, Oude, Nepal, Prome. Por todo ello, se considera que la denominación propuesta por Voigt es la correcta para la variedad, coincidiendo con Isely (1975).

Distribución y hábitat. El origen de la especie y de la variedad candida es algo incierto. Esta última se encuentra citada para India, Burma y China (Trópicos, 2012). En Argentina, en el noroeste y centro, es común en arbolado urbano, también en parques y jardines, siendo menos frecuente que la variedad típica. En Salta, en el límite ArgentinaBolivia, antes de la Aduana y del ingreso al Puente Internacional en Aguas Blancas, se encuentra una población pura y densa de esta especie, que ocupa una superficie estimada de una hectárea, colindante parcialmente con la Ruta nacional $\mathrm{N}^{\circ}$ 50. Los individuos que la componen son de tamaño variable y alcanzan hasta $8 \mathrm{~m}$ de altura y $20 \mathrm{~cm}$ de diámetro, habiéndose observado abundante floración y 
Bol. Soc. Argent. Bot. 48 (3-4) 2013
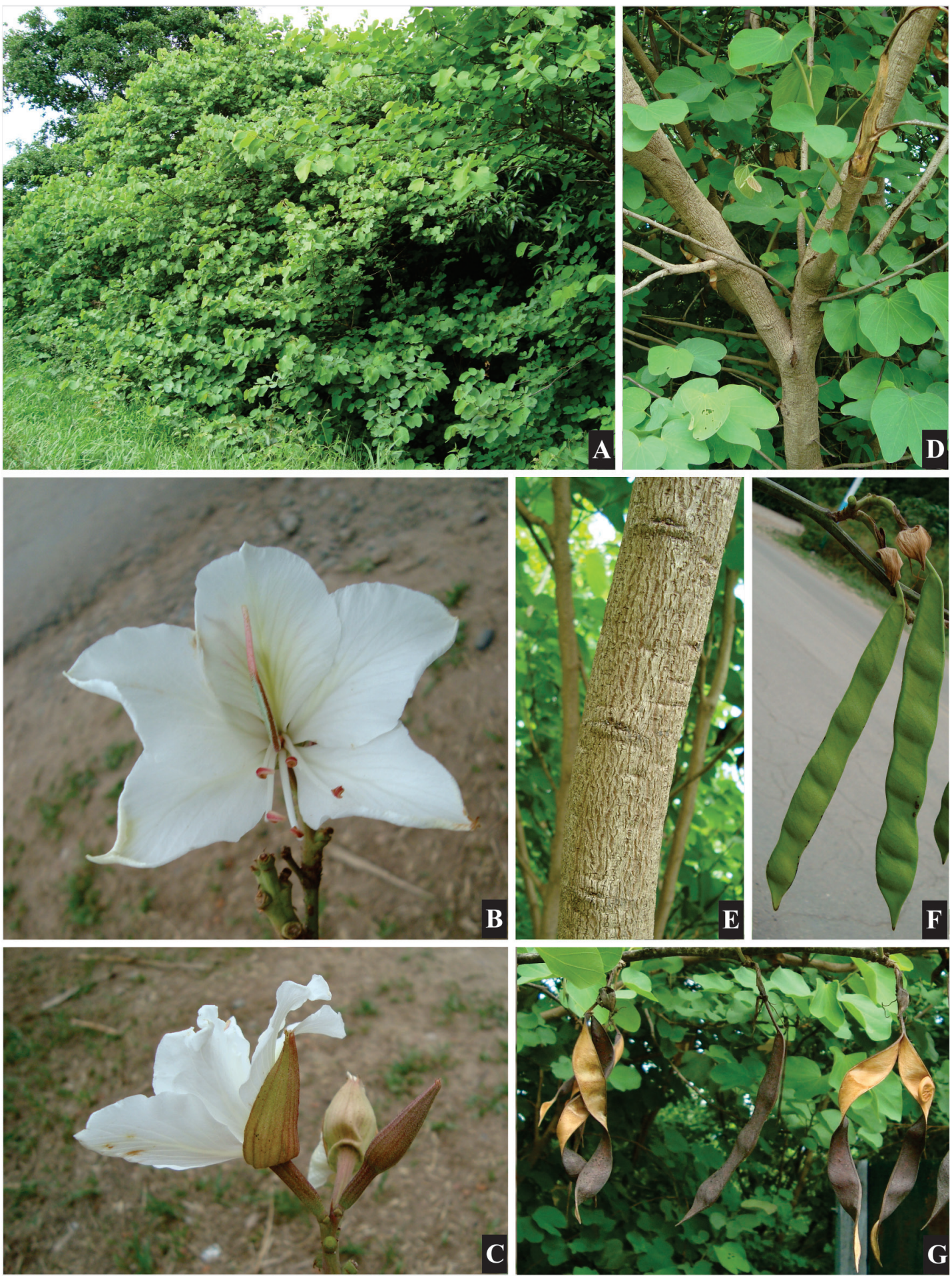

Fig. 2. Bauhinia variegata var. candida. A: Vista de la población en la Selva Pedemontana. B: Flor. C: Flor en vista lateral y botones florales. D: Ramas. E: Tronco y detalle de corteza. F: Fruto inmaduro. G: Población en fructificación plena. Fotografías de E. C. Lozano. 
fructificación. Es probable que su establecimiento se hubiera producido por escape de cultivo desde fincas aledañas (Fig. 2).

Fenología. Se registró para la población estudiada un período de floración de setiembre a noviembre, con fructificación abundante desde octubre hasta diciembre.

\section{Agradecimientos}

Agradecemos a Lourdes Rico (K), Lynda Brooks (LINN), Gweil Lewis (K) y John Hunnex (BM) por la colaboración brindada en la búsqueda de ejemplares tipo en sus respectivos herbarios; y a Sergio Ariel Cáceres (IBONE) por el aporte de bibliografía de difícil acceso para nuestros recursos.

\section{Bibliografía}

BIEGEL, H. M. 1977. Check-list of ornamental plants used in Rhodesian parks and gardens. Rhod. J. Agr. Res. 3: 27.

FORTUNATO, R. H. 1986. Revisión del Género Bauhinia (Cercideae, Caesalpinioidea, Fabaceae) para la Argentina. Darwiniana 27: 527-557.

FORTUNATO, R. H. 1997. Tribu Cercideae. In: A. T. HUNZIKER (ed.), Flora Fanerogámica Argentina 33: 4-9. Museo Botánico de Córdoba, Argentina.
FORTUNATO, R. H. 2002. Bauhinia variegata L. In: J. A. HURREL \& H. B. LAHITTE (eds.), Leguminosas nativas y exóticas. Biota Rioplatense 7: 84. LOLA, Buenos Aires, Argentina.

GAMERRO, J. C. \& R. H. FORTUNATO. 2001. Morfología del polen de las especies argentinas de Bauhinia (Cercideae, Caesalpinoideae, Fabaceae). Ann. Missouri Bot. Gard. 88: 144-158.

ISELY, D. 1975. Leguminosae of the United States: II. Subfamily Caesalpinoideae. Mem. New York Bot. Garden 25: 190-191.

LEWIS, G. P. \& F. FOREST. 2005. Cercideae. En: G. LEWIS, B. SCHIRE, B. MACKINDER \& M. LOCK (eds.), Legumes of the World, pp. 57-67. Kew, Royal Botanic Gardens, England.

SINOU, C., F. FOREST, G. LEWIS \& A. BRUNEAU. 2009. The genus Bauhinia s.l. (Leguminosae): a phylogeny base don the plastid trnL-trnF región. Botany 87: 947-960.

WALLICH, N. 1828[1849]. Numerical list of dried specimens of plants in the Museum of the Honl. East India Company /which have been supplied by Dr. Wallich, superintendent of the botanic garden at Calcutta: 199. Disponible en: http://www.botanicus. org/title/B12082478 (Acceso: Setember 2012).

WIGHT, R. \& G. A. WALKER- ARNOTT. 1834. Prodromus florae Peninsulae indiae orientalis 1, pp. 296. London.

Recibido el 11 de octubre de 2012, aceptado el 20 de mayo de 2013. 
